\title{
Successful Surgical Management of a Tracheo-Innominate Artery Fistula in a Patient with Duchenne Muscular Dystrophy: A Case Report
}

\author{
Ju Sik Yun, M.D. ', Sang Yun Song, M.D., Ph.D. ', Kook Joo Na, M.D., Ph.D. ', Eunchong Kim, M.D. \\ 'Department of Thoracic and Cardiovascular Surgery, Chonnam National University Hwasun Hospital, Chonnam National University School of Medicine, \\ Hwasun; ${ }^{2}$ Department of Thoracic and Cardiovascular Surgery, Chonnam National University Hospital, Chonnam National University School of Medicine, \\ Gwangju, Korea
}

\section{ARTICLE INFO}

Received September 30, 2021

Revised October 28, 2021

Accepted November 10, 2021

Corresponding author

Sang Yun Song

Tel 82-61-379-7663

Fax 82-61-379-7665

E-mail sysong@jnu.ac.kr

ORCID

https://orcid.org/0000-0002-2084-8143
Tracheo-innominate artery fistula (TIF) is a rare, life-threatening complication of tracheostomy that makes it difficult to secure the airway due to massive bleeding, constituting a medical emergency. Therefore, most successful surgical treatments include innominate artery debridement and tracheal fistula repair. Herein, we report a case of successful surgical treatment of a TIF while maintaining cerebral blood flow through an artificial vascular graft.

Keywords: Tracheo-innominate artery fistula, Tracheostomy, Surgery, Case report

\section{Case report}

A 27-year-old man with a tracheostomy presented to the emergency department with hemorrhage in the oral cavity and tracheostomy tube. He had been diagnosed with Duchenne muscular dystrophy at the age of 12 years and had been bedridden ever since. Nine years previously, a tracheostomy was created because of respiratory muscle weakness, and he was placed on mechanical ventilation. Six years ago, he experienced tracheal stenosis due to the growth of granulation tissue inside the trachea, and he underwent a procedure to remove the granulation tissue. Subsequently, an adjustable tracheostomy tube, longer than a standard tube, was inserted to minimize the risk of stenosis recurrence. The patient was followed up at 6-month intervals to evaluate the tracheal condition. On the day of admission, the patient had experienced bleeding for more than 30 minutes before he was brought to our hospital. He immediately received a physical examination by an otorhinolaryngologist; however, no active bleeding was observed through flexible laryngoscopy. Approximately 1 hour after admission, a sudden large hemorrhage was noted in the oral cavity, nasal cavity, tracheostomy tube, and tracheos- toma. Considering the volume of the blood loss, we strongly suspected that the source of bleeding was a tracheo-innominate artery fistula (TIF). At that time, his blood pressure was $80 / 40 \mathrm{~mm} \mathrm{Hg}$ and hemoglobin concentration was $4.3 \mathrm{~g} / \mathrm{dL}$; therefore, saline loading and massive transfusion were initiated. We initially applied external pressure to the suspected fistula site (the upper border of the manubrium), but this was ineffective. Epinephrine-soaked gauze was packed around the cuff of the tracheostomy tube, and pressure was applied around the manubrium using the palm of the hand. Subsequently, the patient's hemodynamic status stabilized, and endovascular interventions were considered. However, we determined that the patient was not eligible for these interventions at our institution; therefore, he was immediately transferred to the operating room for emergency surgery.

We performed translaryngeal intubation with the cuff placed inferior to the tracheostoma. After median sternotomy with neck extension, the proximal and distal ends of the innominate artery were clamped, a TIF was identified (Fig. 1A), and the trachea was separated from the innominate artery. Primary repair of the artery was deemed difficult; therefore, graft interposition of the innominate artery 

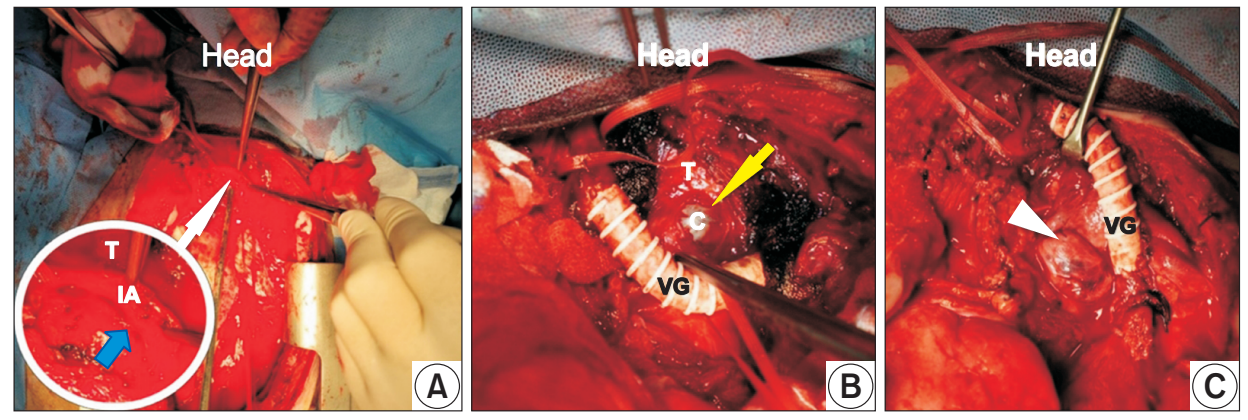

Fig. 1. Intraoperative findings. (A) A tracheo-innominate artery fistula (blue arrow). (B) The tracheal defect (yellow arrow) was exposed after graft interposition of the innominate artery. (C) A pedicled thymus flap (arrowhead) was observed between the vascular graft and the trachea. $T$, trachea; IA, innominate artery; $V G$, vascular graft.
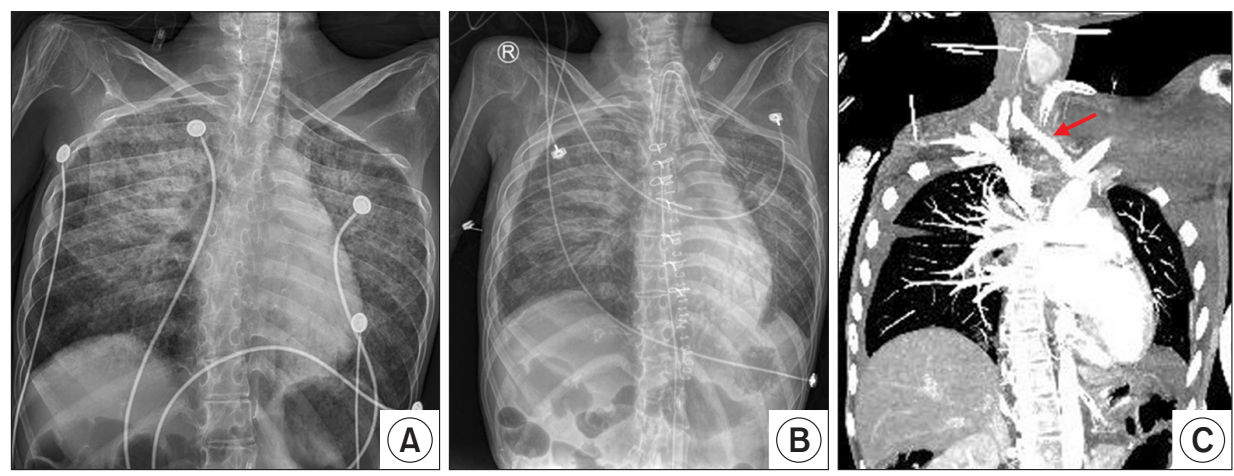

Fig. 2. (A) Preoperative chest radiograph shows features suggestive of aspiration pneumonia. (B) Postoperative chest radiograph. (C) Postoperative computed tomography angiogram. The red arrow indicates the interposed vascular graft.

(Impra ePTFE Vascular Graft $8 \mathrm{~mm}$; BD, Franklin Lakes, NJ, USA) was performed to maintain flow to the right carotid and subclavian arteries. A defect measuring approximately $2 \mathrm{~cm}$, along with granulation tissue, was noted on the anterior wall of the trachea (Fig. 1B). The granulation tissue was removed after the trachea was released proximally and distally. Subsequently, primary repair of the tracheal fistula with interrupted sutures was performed. To prevent irritation of the anterior tracheal wall due to the vascular graft, a thymus flap was placed and fixed between the reconstructed innominate artery and the trachea (Fig. 1C). A conventional tracheostomy tube was inserted postoperatively, and the cuff was not inflated to avoid irritation of the tracheal mucosa. The patient's condition was monitored in the intensive care unit for 3 days, after which he was transferred to the general unit (Fig. 2). Antibiotics were administered for 10 days to treat pneumonia, and there were no specific findings on neurological examinations. Three weeks later, the patient was in a stable condition and was transferred to another hospital. Long-term follow-up is required to confirm the effectiveness of this surgical technique. The patient's family provided written informed consent for the publication of his clinical details and images.

\section{Discussion}

TIF is a rare but fatal complication of tracheostomy, with an incidence of $0.1 \%-1 \%$ after surgical tracheostomy $[1,2]$. TIF has been reported to develop after percutaneous dilatational tracheostomy [3]. Patients with TIF usually have poor outcomes because TIF is a sudden-onset medical emergency that involves a large volume of blood loss and difficulty accessing the airway. TIF is characterized by massive arterial bleeding through the tracheostomy tube, and its diagnosis is mainly based on clinical suspicion [4]. Assessment using bronchoscopy or laryngoscopy can be helpful if the bleeding is controllable or not excessively severe. Additionally, computed tomography angiography of the neck, including the aortic arch, can aid in making the diagnosis.

When TIF is suspected, appropriate emergent treatment is essential. Maintaining hemodynamic stability through the termination of bleeding and rapidly securing the airway followed by adequate ventilation is important. To achieve this, one can place an overinflated tracheostomy tube cuff at the suspected bleeding site or manually compress the innominate artery against the posterior sternal table after enlarging the tracheostoma [5]. After emergent treatment, the TIF itself should be treated.

The treatment guidelines have not been clearly estab- 
lished because TIF is a rare and fatal condition. To date, emergency surgery is the most commonly performed treatment, and there have been many reports of endovascular interventions in recent years. Surgery is performed through a right collar incision and upper median sternotomy or standard median sternotomy with neck extension. The location of the TIF is identified after exposing the branches of the aorta and the trachea. To manage bleeding, most surgeons choose to divide the innominate artery after arterial suturing or ligation. Significant neurologic or vascular compromise has rarely been reported when this approach is used. Furukawa et al. [4] monitored the cerebral blood flow of patients undergoing division of the innominate artery by measuring the blood pressure of the bilateral radial arteries or measuring the regional cerebral oxygen saturation using near-infrared spectroscopy and reported that no significant laterality was noted in 6 of 7 patients. Other techniques of treating TIF include primary repair, bypass of the innominate artery to the right common carotid artery, and jugular or saphenous vein/artificial vascular graft interposition. As TIF is often an emergency, the surgical technique should be carefully selected for each patient after considering the risk of complications, such as recurrent bleeding, infection, stenosis, and tension on the vascular anastomosis.

Direct closure is the first choice of treatment for tracheal fistula repair, although some reports suggest using a pericardial or thymus flap to repair large defects [6]. Sternocleidomastoid or strap muscle flaps or pedicled thymus flaps are used to separate the trachea from the artery. In the case described herein, artificial vascular graft interposition was used to decrease the duration of surgery and to maintain adequate cerebral blood flow. We believed that the graft might irritate the anterior tracheal wall; therefore, we placed a pedicled thymus flap between the trachea and the artery.

In recent years, there has been an increasing number of reports on endovascular interventions. Taechariyakul et al. [7] reported a case of TIF treatment and conducted a literature review with a pooled cohort analysis. They found that endovascular stent grafting $(n=33)$ had comparable postprocedural survival rates and lower complication rates than open surgical repair $(\mathrm{n}=137)$ [7]. In their systematic review, which included 27 patients with TIF who received endovascular interventions, O'Malley et al. [8] found that endovascular intervention may be a feasible alternative to sternotomy and suggested that this approach may be useful in poor surgical candidates. However, a prompt endovas- cular intervention can only be performed if the patient is hemodynamically stable, adequate instruments are available, and there is an experienced radiologist.

Here, we report a case of successful surgical management of a TIF in which graft interposition was used to maintain adequate cerebral blood flow. We hope that the details of this case will be helpful in establishing guidelines for the emergency management of this rare condition.

\section{Conflict of interest}

No potential conflict of interest relevant to this article was reported.

\section{ORCID}

Ju Sik Yun: https://orcid.org/0000-0002-5167-3454

Sang Yun Song: https://orcid.org/0000-0002-2084-8143

Kook Joo Na: https://orcid.org/0000-0003-0923-1414

Eunchong Kim: https://orcid.org/0000-0002-1119-4125

\section{References}

1. Allan JS, Wright CD. Tracheoinnominate fistula: diagnosis and management. Chest Surg Clin N Am 2003;13:331-41.

2. Scalise P, Prunk SR, Healy D, Votto J. The incidence of tracheoarterial fistula in patients with chronic tracheostomy tubes: a retrospective study of 544 patients in a long-term care facility. Chest 2005; 128:3906-9.

3. Grant CA, Dempsey G, Harrison J, Jones T. Tracheo-innominate artery fistula after percutaneous tracheostomy: three case reports and a clinical review. Br J Anaesth 2006;96:127-31.

4. Furukawa K, Kamohara K, Itoh M, Morokuma H, Morita S. Operative technique for tracheo-innominate artery fistula repair. J Vasc Surg 2014;59:1163-7.

5. Ailawadi G. Technique for managing tracheo-innominate artery fistula. Oper Tech Thorac Cardiovasc Surg 2009;14:66-72.

6. Draeger TB, Andaz SK, Gibson VR. A novel reconstruction technique of a tracheal defect in the emergent setting using a thymus flap in a patient with tracheoinnominate artery fistula. Surg Case Rep 2020;6:21.

7. Taechariyakul T, Keller FS, Jahangiri Y. Endovascular treatment of tracheoinnominate artery fistula: case report and literature review with pooled cohort analysis. Semin Thorac Cardiovasc Surg 2020;32: 77-84.

8. O'Malley TJ, Jordan AM, Prochno KW, et al. Evaluation of endovascular intervention for tracheo-innominate artery fistula: a systematic review. Vasc Endovascular Surg 2021;55:317-24. 